\title{
Advances in telecare over the past 10 years
}

This article was published in the following Dove Press journal:

Smart Homecare Technology and TeleHealth

12 November 2013

Number of times this article has been viewed

\section{Kenneth J Turner' Marilyn R McGee-Lennon² \\ 'Computing Science and Mathematics, University of Stirling, Stirling, UK; \\ ${ }^{2}$ School of Computing Science, University of Glasgow, Glasgow, UK}

\begin{abstract}
This article reviews advances during the past decade or so in telecare (ie, computersupported social care at home). The need for telecare is discussed along with how it relates to social and health care. The expected benefits of telecare are also discussed. The evolution of telecare technology is reviewed, covering various system generations. The capabilities of present day telecare are covered, along with its advantages, limitations, and barriers to uptake. Recent evaluations and exemplars of telecare are discussed. The user requirements for telecare are presented, complemented by a discussion of the issues in user and professional acceptance. The article concludes with a summary of past developments in telecare and the prospects for the future.
\end{abstract}

Keywords: assisted living, health care, home care, independent living, social care, telehealth

\section{Introduction Context}

The world population is aging, with the percentage of older people (over 65) gradually rising. In the UK, for example, this percentage was $24.4 \%$ in 2000 and is expected to become $39.2 \%$ by $2050 .{ }^{1}$ In Europe, the number of older people is expected to grow from 75 million in 2004 to 133 million in $2050 .^{2}$ A similar situation applies in other developed countries, with much higher percentages forecast for some areas (eg, 71.3\% by 2050 in Japan).

Clearly, this will increase the need for care of older people. Although people are living for longer, many have to deal with long-term, age-related conditions. The growing percentage of older people, coupled with increasing pressure on social and health care budgets, means that care providers will be increasingly challenged to cope with continuing delivery of care. As a result, it will not be feasible to provide sufficient care homes and hospital stays (which are much more expensive than looking after someone in their own home). There is an increasing need to change the way we think about and deliver care services.

\section{Social and health care}

Social care supports the wellbeing of individuals in the community, while health care deals mainly with the diagnosis and treatment of illness and impairment. An important part of social care is helping older people to live independently in their own homes and communities. This covers a range of factors in social and mental wellbeing including 
activities of daily living (ADLs). ${ }^{3}$ Basic activities include eating, hygiene, and mobility, while instrumental activities include communication, housework, and shopping. As a person gets older, these may become more difficult to achieve or maintain due to physical or mental deterioration.

Social care and health care have traditionally been supported by different organizations and funded separately. However, there are benefits in bringing social and health care closer together. Both concern the wellbeing of the individual. Social problems can have an impact on health. For example, a stay-at-home individual could become withdrawn, might miss out on regular exercise, and may fail to eat properly. Health issues can also adversely affect social behavior and wellbeing. For example, a health condition may lead to the individual missing out on social contact, not engaging with the community, and becoming depressed.

Fortunately, there is now a trend toward more integrated and holistic care. As an example, the Single Shared Assessment ${ }^{4}$ performed in Scotland allows social and health care needs to be evaluated. As a result of a care assessment, technologysupported solutions for both may now be prescribed. There are also possibilities for commonality in facilities to support social and health care. For example videoconferencing might be used for keeping in touch with the community and also for remote health consultation, while a home hub might collect and forward both social and health care data. Clearly, the line between social and health care has become blurred over the last decade, and this has had a significant impact on thinking about telecare and telehealth solutions. This can be seen, for example, in the shift away from talking about telecare and telehealth as distinct technologies with different aims. Now, the emphasis is more on independent living technologies and life-enhancing technologies - umbrella terms encompassing a blend of telecare, telehealth, and mainstream technologies that support wellbeing and health.

\section{Telecare}

Technology for home care has been enthusiastically embraced as part of the solution for the aging population. Telecare refers to automated support of social care at home. This includes monitoring for potentially harmful situations (eg, falls, overflowing sinks or baths, or night wandering) as well as a range of services for people with physical impairment or mobility issues (eg, curtain openers, door entry phones, and home automation). Telehealth (also called telemedicine or e-health) refers to remote support of health care at home. This includes remote consultation and diagnosis (typically via videoconference) as well as monitoring health parameters and vital signs (eg, blood pressure, heart rate, and seizure risk) which can be sent via the Internet to a general practitioner, nurse, or clinic.

Although "telecare" is the generic term used in this article, other terminology is also used in this field. As social and health care are becoming increasingly intertwined, the term "telehealthcare" has been used in the UK to mean remote home care support. Assisted living technologies and independent living technologies refer to solutions that help older people to prolong an independent life at home. Assistive technologies are more general, being all kinds of devices that help with daily living, such as wheelchairs and stair lifts. Home automation aims to improve management of the home (eg, appliance control, entertainment, and heating), while building control refers more to an office environment. Smart homes provide a degree of intelligence and programmability. Ambient intelligence refers to the automated capabilities of a smart home.

Home care systems are computer-based systems that support delivery of care..$^{5}$ Typically, some kind of home hub is provided to collect, analyze, react to, and forward care data collected from a variety of sensors or other input devices. Besides sensors for inputs, a home care system can use software services (eg, for communication, speech input/ output, or weather forecasts). A home care system is able to respond through a variety of actuators to control appliances, maintain the home environment, signal alert conditions, for example. More sophisticated systems have a degree of programmability, allowing customization for individual user needs and adaptation to changing circumstances.

Home care technologies offer significant benefits. Particularly in rural settings, the ability to support care at a distance can save substantial travel. For this reason, many health authorities are promoting self-care at home rather than relying exclusively on centrally provided care. Trends, anomalies, and alert conditions can be identified and reported to a central location (eg, a call center or a health center). Family members can be reassured that the user is being monitored for undesirable situations. Professional carers can also be relieved of low-level monitoring tasks.

Telecare aims to provide computer-based support for these kinds of activities. ${ }^{6}$ At the minimum, this involves monitoring the extent to which people are living normally at home. For example, nonintrusive sensing can confirm that the individual is sleeping well, is active around the house, and is dealing with personal hygiene and toileting. A telecare system will also typically check for potentially hazardous situations such as a gas cooker not being lit, water being left 
running, or the user falling. More advanced systems can help with activities through speech-based or visual prompting, and by reminders such as for medication and appointments. Trends and anomalies can be noticed in the user behavior and reported to carers or to the users themselves for further investigation. $^{7}$

Recent projects have addressed the need to empower users themselves by providing methods for people to monitor and visualize their own activities and data. This allows people to make proactive and preventative care and lifestyle decisions themselves. Older people can therefore be assisted to stay longer in their own homes, where they are in familiar surroundings and near to the people and the area they know.

\section{History and development of telecare Evolution of telecare}

Telecare technology is said to have gone through three generations. ${ }^{8}$ The first generation of reactive telecare systems mainly focused on social alarms. For example, users could use a pendant to signal a call center for help or to contact support staff. The second generation of proactive telecare systems allowed more automatic responses based on sensor information. For example, a fall detector could automatically report an alert condition without the need for user intervention. The third generation of integrated systems is aimed at enhancing the user's quality of life. For example, virtual communities can link the user into a wider care network and can provide access to remote services for communication and advice.

There is not yet an agreed framework or set of standards for building telecare systems. The European Telecommunications Standards Institute Special Task Force 264 is working on standards for telecare. ${ }^{9}$ Although more focused on telehealth, the Continua Health Alliance is developing standards for interoperability in home monitoring. ${ }^{10}$

\section{Telecare technologies}

The context of a sample telecare system is shown in Figure 1. Sensors within the home are typically connected wirelessly (radio or infrared) as this minimizes disruption due to additional wiring. However, in new-build housing there may already be suitable wiring in place (eg, Cat 5/6 cables or a proprietary home automation installation). Actuators may also be connected wirelessly or through existing wiring (eg, the $\mathrm{X} 10$ standard for mains appliances). ${ }^{11}$ Other standards such as $\mathrm{UPnP}^{12}$ (Universal Plug and Play) use computer networks like Ethernet, ${ }^{13}$ Bluetooth, ${ }^{14}$ or ZigBee. ${ }^{15}$

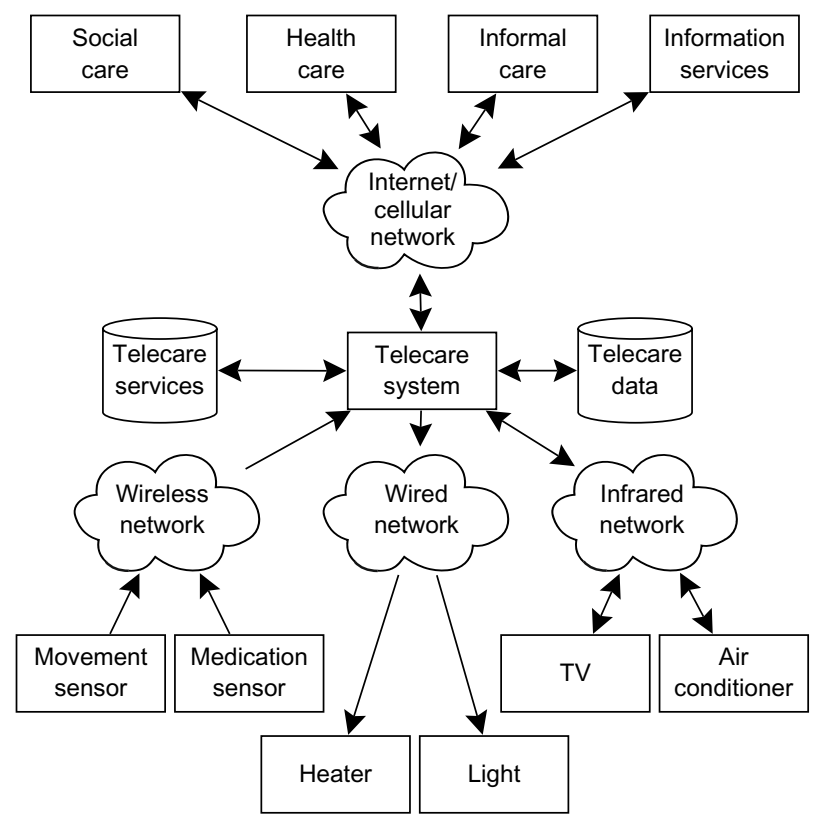

Figure I Sample telecare system in context.

Note: Reprinted from Turner KJ, Maternaghan C. Home care systems. In: Turner KJ, editor. Advances in Home Care Technologies: results of the MATCH project. Amsterdam: IOS Press; 201 2:21-29.

A telecare system collects behavioral and environmental data from sensors within the home. This information is stored locally, for possible analysis or summarizing prior to uploading to a care center. Care services react to this data to ensure the user's safety, to provide reminders, to look after the home, for example.

Originally telecare systems used a phone line for uploading data securely and for communication with a call center. It is now common to connect using a broadband or cellular network. For telecare, user data can be presented locally (eg, for use by a carer) or can be sent to a social work center. With the user's agreement, alerts and high-level information can also be sent to informal carers (eg, family and neighbors). In recent years, there has been a movement toward providing some of this data to users themselves to promote increased independence and self-directed care. Some kinds of telecare data with health implications (eg, diet, medication, or sleeping) can be sent to a health center. Given an Internet connection, external services can also send useful information to the home (eg, community communication or weather forecasts).

Early base units for telecare were designed with custom electronics. However, for some time it has been normal for home hubs to use an embedded computer system. The home hub might also be a set-top box or a standard PC (personal computer). The power of smart phones means these are suitable as the home hub and as a source of sensor information. 
With computer-based systems, telecare can exploit standard computing facilities such as communication, networking, and programmability.

At first, telecare sensors were relatively unsophisticated such as pendant alarms, flood detectors, and gas monitors. Devices for home security and home automation such as movement detectors, occupancy sensors, and door switches were then adapted for use in telecare. Over time, the range of sensors has expanded to include quite sophisticated ones such as fall detectors, seizure monitors, and medicine dispensers. Telecare actuators also started out fairly basic, eg, water or gas shutoff valves. Again, home security and home automation actuators have extended what is provided for telecare. For example, home appliances can be controlled wirelessly or through mains wiring, while door entry phones and curtain motors help those with a physical disability.

More recent telecare devices have shown a trend toward mobility and different sensory modalities. Data can be collected from sensors worn around the body in what are called body sensor networks. ${ }^{16}$ Mobile sensors have also been used, for example to help people with mental health problems. ${ }^{17}$ Multimodal interfaces allow users to interact with a telecare system in ways that suit the person's needs, preferences, and environment. ${ }^{18}$ For example, audio and speech are alternatives to visual communication. Touch and gestures can also be used, and even smell can be useful - say, to remind the user to cook a meal.

For research projects at least, ${ }^{5}$ a popular solution for home hubs is OSGi (originally Open Services Gateway initiative). ${ }^{19}$ OSGi is described as a dynamic module system for the Java programming language. The software components called bundles are modular and self-contained. An OSGi system can readily be made extensible by configuring bundles, allowing capabilities to be added or modified while the system runs. Bundles can communicate through events, allowing them to remain loosely coupled. OSGi scales well and can run on many kinds of system from small embedded systems to desktop computers.

Telecare can be only as good as the data it works with. Physical measurements (eg, for bed occupancy, appliance use, or blood pressure) are generally reliable and have known accuracy. Other sensor data (eg, for a fall, a seizure, or a spoken command) may be more challenging to collect accurately. Less certain data can therefore be augmented in other ways. For example, accelerometer data that suggests a fall might be confirmed by a video image that shows the user lying on the floor. Where there are multiple occupants in the home, such as the user receiving telecare and a partner, it can be difficult to distinguish data from the cared-for person and from someone else in the same home. Again, multiple sources can increase the reliability of the data.

Telecare can collect large volumes of information. The difficulty is then to sift through this information in order to draw meaningful conclusions. In fact, the low-level data is often not of interest. What matters is the high-level conclusions that can be drawn from it. A common approach is to use the data to build models of the user behavior. This can then be analyzed for trends and anomalies (eg, poor sleep patterns or restlessness), and can be presented in a way that is meaningful to carers. ${ }^{20}$ In general, analysis and interpretation of care data has received considerable attention. ${ }^{21}$

There is a growing body of research into how users can be supported in the self-management of a chronic condition or their general wellbeing. ${ }^{22}$ Clinicians and computer scientists are investigating the type of interfaces and the types of visualizations (such as graphs of symptoms or activity) that people need to monitor and successfully reflect on their health and wellness over time. ${ }^{23}$ Social scientists are also exploring this from a behavioral modification point of view. The aim is to understand better how telehealth and telecare can actually motivate change in behavior based on feedback from telecare devices. ${ }^{24}$

\section{Telecare services}

A variety of telecare services offer automated and computerbased support for care in the home. The Center for Aging Services Technologies categorizes these systems into three broad domains: safety, health and wellness, and social connectedness. ${ }^{25}$ The following examples give an idea of the range of services that can be provided.

\section{Safety}

Potentially risky situations can be monitored and managed. For example, a flood detector can report an overflowing sink or bath in case the user leaves the faucet running. A gas or smoke detector can similarly alert the user or a carer to a problem, turning off the gas supply if a cooker is not lit. Falls are a serious problem for some older people, so a fall detector can be beneficial.

\section{Security}

The system can support peace-of-mind in the home. When the user leaves the home unoccupied, doors and windows can be automatically locked if necessary. If there is movement in an unoccupied house, an alert might be sent about a possible intruder. While the user is on holiday, curtains might 
be closed at night and lights might be turned on to give the impression of occupancy.

\section{Behavior}

Monitoring technologies can track general ADLs, instrumental ADLs, and other behaviors. This can be achieved with wearable activity monitors (accelerometers and sensors) as well as fixed activity monitors. If the user sleeps poorly, information about restless nights may be of value to a carer. A user may be prone to waking at night thinking that it is time to go out (called night wandering). Gentle encouragement from the system to go back to bed may be necessary in these circumstances. If the user forgets to make meals, the system can give reminders about when a meal is due.

\section{Communication}

The system can support digital communication to/from the home, allowing the user to stay in touch with friends, family, and carers. Basic communication facilities include email and (text) messaging. Other forms of communication include videoconferencing (for informal contact or remote consultation) and community television (for keeping in touch with the neighborhood).

\section{Entertainment}

The system can support entertainment within the home. If a user is forgetful, the system can note which television programs the user normally watches and issue reminders about these (or automatically record them). If the user likes to listen to music at certain times, the system can learn the user's preferences and play music as appropriate (eg, when the user comes home or is cooking). The system can also support (multi-player) games that encourage exercise and social communication.

\section{Home automation}

The system can automate various home functions on behalf of the user. Environmental conditions can be controlled to ensure that each room has the desired temperature, humidity, and light level. For someone who is not very mobile, the system can deal with the curtains or can open the front door to a recognized caller. If codes or tags on household goods are scanned, the system can monitor stock levels and automatically reorder items that are getting low. The system can also suggest recipes based on what is available.

\section{Uptake of telecare technologies}

A growing range of telecare solutions is becoming available but uptake is still relatively low. There is a pressing need to understand why and to find out how to change this. Governments in most developed countries have recognized the issue of an aging population and have put telecare programs in place to stimulate growth in this area.

Scotland took early steps to deploy and evaluate telecare equipment. ${ }^{26}$ This was followed by the Preventative Technology Grants for telecare in England, with corresponding grants in other parts of the UK. Numerous pilot studies of telecare have been conducted in the UK, but the largest so far has been the Whole System Demonstrator program. ${ }^{27,28}$ Also in the UK, the Assisted Living Innovation Platform program and its successor program DALLAS (Delivering Assisted Living Lifestyles At Scale $)^{29}$ have been designed to evaluate telecare technologies at scale through the involvement of many users.

As examples from elsewhere in the world, various framework programs in Europe have supported telecare research. The Ambient Assisted Living program has also been active in Europe since $2007 .{ }^{30}$ In the USA, major corporations such as Cisco, Google, IBM, Intel, and Microsoft have worked on telecare and telehealth solutions. The Veterans Association has reported extensive experience of telehealth and telecare. ${ }^{31}$ As noted earlier, Japan faces a particularly severe challenge due to a high proportion of older people. Despite strong support for telecare in Japan, the results so far have been fragmented..$^{32}$ However, Japan has made particular advances in robotic assistance for care.

Telecare has been demonstrated to offer social and economic benefits, though the savings are more through efficiency gains than actual cash savings. ${ }^{33}$ From the point of view of end users (the residents receiving care), telecare offers convenience and peace of mind. Informal carers such as family members also appreciate the reassurance that a telecare system offers. Formal carers are relieved of routine monitoring. Care providers also anticipate lower costs due to reduced demand for hospital beds and fewer unplanned hospital admissions. However, the results of the Whole System Demonstrators in England suggest that the benefits of telehealth may not be so clear cut. ${ }^{27,28}$

Telecare cannot, of course, replace human care. Even if large amounts of sensor data can be collected, interpreting this in a meaningful fashion requires human judgment. ${ }^{34}$ There is even a risk that users will place undue reliance on a telecare system. End user acceptance of telecare is generally positive, but more attention is needed to good design. In Europe, at least, a common concern is that telecare will invade privacy. ${ }^{35}$ In contrast, telecare in the USA is perceived to be part of telemedicine and so raises fewer concerns about it being "state surveillance." 36 
The MATCH project (Mobilising Advanced Technologies for Care at Home $)^{37}$ conducted a qualitative study on the barriers to the uptake of home care technologies ${ }^{38}$ This included a variety of stakeholders in telecare, including older users, their friends and family, health and social care professionals, and policy makers. This study found that the main barriers to the uptake of telecare in the UK were:

- Availability of resources

- Awareness raising and education

- Acceptance issues

- Personalization and evolution of services

- Ethical and legal issues.

It is clear from the initiatives discussed above that funding and deployment of telecare are increasingly being supported. Lack of awareness among care providers, however, has been a key barrier to uptake of telecare. ${ }^{38}$ This situation is gradually improving. Most social and health care professionals were trained at a point that telecare was in its infancy. It will take time to train a new generation of practitioners and to retrain existing ones in telecare technologies. Telecare will need to be incorporated into daily care practices and lifestyles, forming one of a range of solutions that can be delivered. ${ }^{39}$

A later section discusses acceptance of telecare technologies and how technologies and services can be personalized for the user. Ethical issues (eg, security and privacy of care information) are complex. They cannot be covered in detail in this article, but are addressed briefly later. Although there are some encouraging results from telecare pilots, a robust cost-benefit analysis and evidence base for telecare at scale still needs to be established when telecare is deployed in people's homes over time. However, various organizations have begun collecting relevant data for this..$^{29,40,41}$ An overview is given later of some leading telecare evaluations.

\section{User perspectives}

There has been a significant cultural change over the past decade in the way that care services are thought about. For example, models of health care have moved from clinician-centric to patient-centric and now, more recently, to consumer-centric. In addition, there are several initiatives to integrate health and social care, providing a more joined-up health and wellness care service. This is a significant cultural shift that makes it even more crucial to consider the attitudes and opinions of a range of stakeholders in care in order to design and deliver telecare that will be successful.

\section{Telecare stakeholders}

The design of new home care technologies such as telecare needs to consider the wide variety of people who might use the technology. These users may be directly or indirectly interested in the data that the technology collects or produces regarding health and wellbeing. Often, the users of telecare are older adults with varying needs and capabilities. Telecare has to be designed to support an older adult who may have problems of declining vision, hearing, or physical mobility. Devices themselves have to take this into account and be designed to be usable and acceptable yet not stigmatizing. This balance is often difficult to achieve. More advanced telecare systems can compensate by making the information available via multiple sensory channels (eg, speech reminders for someone with a significant vision impairment). ${ }^{42,43}$

In addition to the person being cared for in the home, other people involved in care are likely to include the following who are all stakeholders in home care technology:

- Partners living in the same home

- Friends and family who are involved in care or interested in its status

- Visiting care professionals such as occupational therapists and community nurses

- Remotely located care staff such as social workers and consultants.

Individuals with telecare equipment in their home are direct end users. This can include older adults, people with impairments (sensory, physical, or cognitive), and those with a long-term condition for whom telecare can offer assistance. Friends, family, neighbors, and informal carers are potential end users, as they may interact with the equipment. They are also likely to be interested in what the technology does, perhaps to check the wellbeing of an older family member. Care professionals too are obvious users as they have a stake in the home care data collected (eg, about deterioration in sleep patterns or activity around the home). Each of these categories of telecare users can be active or passive.

An active user interacts directly with the equipment to input data (eg, when medication is taken) or to use outputs from the system (eg, a graph of weekly activity in the home). A passive user of telecare has equipment that monitors home activity (eg, a fall detector) and alerts a call center or family member (rather than the occupant) when intervention of some kind is required. There has been a significant move in recent years from passive monitoring to more active use of telecare technologies that allow and encourage direct interaction. This change is due in part to older adults being more computerknowledgeable. Another reason is the increasing shift in 
the balance of care, empowering users to manage their own health and wellbeing.

There are several key indirect stakeholders in telecare, including:

- Designers and manufacturers of the equipment and its interfaces

- Technicians who set up and maintain the equipment

- Call center staff who respond to alerts

- Health and social care professionals who prescribe and champion telecare

- Policy makers who shape the way telecare is delivered and promoted.

A growing number of people are likely to interact with telecare and the increasing variety of technology interfaces (eg, to view data). As a result, there is an increasing need to improve the computer interfaces and physical devices themselves. Several key factors are shaping the way telecare is (or is not) being used; the following section discusses some of these issues.

\section{Usability and user experience}

Making services usable and acceptable is vital if telecare technologies and services are to be taken up and to be successful in practice. If the equipment is not usable, it will end up tucked away in a drawer rather than being used fully. As well as designing new functionality (eg, new sensors) and new ways to interact with telecare (eg, gesture and speech input), interfaces and devices also need to be more configurable for the variety of user needs and preferences. Consumers should want to purchase and use telecare, and to have it in their homes for a length of time. Users, plus their family and friends, need to be comfortable with what data is being collected and with how this data is used for positive gain - to provide reassurance or to avoid a hospital stay for example. Telecare systems also need to provide control where people want it, and yet to fade into the background where preferred. To promote the potential of telecare, the technology must be made more desirable and less stigmatizing. It must also be able to offer a rich experience without disrupting the user's home or life significantly. More work is needed on how to improve the overall usability and user experience of telecare technology.

The MATCH project ${ }^{37}$ has established significant results about the perceptions of telecare technologies among a wide range of stakeholder groups. ${ }^{38}$ Projects like COBALT (Challenging Obstacles and Barriers to Assistive Living Technologies $)^{44}$ have looked at the perceived barriers for uptake of assisted living technologies and how they can be made less stigmatizing, more usable, and more acceptable to older adults. Projects like MultiMemoHome ${ }^{42}$ have looked at co-designing technologies with older adults and developing reminder systems that are personalized for user needs and preferences.

The following is a brief overview of some of the current barriers to the uptake of telecare and how these might be overcome in the future.

\section{Design}

The visual look and feel (or esthetics) of telecare products are slowly improving. Telecare needs to have clear displays (since visual acuity declines with age), but there is no reason why it should be stigmatizing or look like a piece of "care equipment." Instead, devices need to be attractive to the consumer and fit in with the décor of the home. Product designers are working on the DALLAS program to coproduce services and technologies that consumers say they need. ${ }^{29}$ This includes working on branding and consumer awareness rather than seeing telecare purely as a necessity.

\section{Functionality and interaction}

There is still work to be done on making telecare devices that people can easily interact with, and also readily configure to their own needs should they choose to. This will require enhanced functionality as users become more familiar with the systems, and will also require user interfaces that adapt to the individual's needs and capabilities over time. Users of telecare are often older adults and people with physical, sensory, or learning impairments. Attention is therefore needed to making telecare technologies more acceptable to a wide variety of age ranges, expertise, capabilities, and preferences. The design should cope with multiple users (of the system and the data it generates), and must provide functionality appropriate to each user. More and more telecare systems are providing hubs or displays so that the user can configure the system to behave in a particular way, can view their own health or wellbeing data, and can share this information with friends, family carers, and health professionals where appropriate. Users and carers prescribing telecare need to be aware what functionality is being provided and what functionality is appropriate for each individual.

\section{Personalization}

Until now, telecare technologies have tended to be closed and standalone point solutions. There have been many recent proposals that telecare should be more easily personalizable for users (by themselves or by a carer). ${ }^{38}$ This would increase 
the reach of technology, making it more accessible to people with physical and sensory impairments, and could also help the technology to fit more seamlessly into a person's lifestyle and therefore to increase acceptance. However, the interfaces required for personalization need further research. Simple interfaces are required that allow novices to easily pick which types of interactions with the system are appropriate for them (eg, deciding that a reminder should be sent to a cell phone rather than to a television screen). Different users need different levels of visibility and control, so this also has to be taken into account.

\section{Ethics and privacy}

Ethical and privacy issues continue to drive what is permissible and acceptable in the field of telecare. The technology should achieve a balance between being unobtrusive yet not entirely hidden from users. As early as 1997, Fisk ${ }^{45}$ suggested that an ethical framework was required for telecare. More recently, Clark and McGee-Lennon ${ }^{38}$ identified that there are still concerns about privacy and ethics among not only end users but also care professionals and policy makers. Some of the common themes that have been identified are:

- Fear about the technology failing

- Accountability when technology negatively impacts a person's health or wellbeing

- Worry about infringing on the privacy of the individual

- Concern over who has access to confidential care data

- Uncertainty over the security of data being sent to and from the home

- The varied capacity of the individual to provide informed consent.

There are varied perceptions surrounding what data is being collected, who owns and controls the data, and what is being done with the data. Until care professionals and end users are clear about some of these issues, the acceptability of telecare will continue to be limited. Ethical and legislative issues will clearly need to continue to evolve.

\section{Awareness raising and championing}

One of the most prevalent findings among care professionals and policy makers in the past decade has been that there is a clear demand for awareness and knowledge of the range, scope, and capabilities of assistive and home care technologies. This may simply require awareness raising using up-to-date leaflets and training material. A more indepth approach may also be needed to cover the assessment protocols and procedures that exist within care organizations. Many governments are introducing telecare champions and demonstrator "smart homes" to educate people about what newer generations of technology can and cannot do in the context of telecare.

Examples of the information needed include:

- What technology is available

- The advantages and disadvantages of particular equipment

- Prescribing technology that is actually needed rather than "one-size fits all" packages

- Knowledge of where and how to acquire technology

- Awareness of organizational funding procedures

- Knowing what permissions are required (eg, informed consent and other ethical aspects)

- Being aware of the privacy and security issues due to increased home connectivity

- Understanding how client preferences and situations affect acceptability

- Knowing how to evaluate actual use of the technology.

\section{Acceptance}

All of the above factors can influence the overall likelihood of telecare acceptance. In the past, telecare has sometimes been seen as stigmatizing. Many older adults do not use pendant alarms, for example, as they are seen as a sign of getting old or needing help. Acceptance levels may also vary depending on the social context and the amount of technological knowledge a stakeholder has. The perceived benefit of telecare equipment has to be clear before it becomes acceptable in someone's home. If the equipment is designed well, and if using it allows the user to live more independently, then acceptance should increase dramatically.

\section{Costs and care budgets}

Cost is an additional challenge and potential barrier to the wider rolling out, scaling up and mainstreaming of telecare. With care budgets in most countries currently being stretched, telecare delivery must be affordable for the end user and also offer savings in social and health care budgets. Who pays for the technology is an ongoing debate in most countries. Even in the UK, National Health Service models include a more consumer-oriented approach. End users are encouraged to buy mainstream devices off the shelf from their own personal budget, and then purchase telecare services from their personal care allowance. Further complications arise when trying to establish a true cost-benefit analysis. Health and social care budgets are often separate, so it can be difficult to establish where the cost savings actually are. For example, a telecare monitoring service could be supplied 
by social services but reduce the number of days spent in a hospital bed. Further work is clearly needed on defining sustainable business cases for the larger scale deployment of telecare. This is something the DALLAS program in the UK is looking at in more detail. ${ }^{29}$

\section{Evaluations of telecare}

Evaluation is vital in progressing the rollout, uptake, and general acceptance of telecare technologies and services. Previous evaluations have tended to be small-scale and somewhat fragmented pilot studies. It has become generally accepted that telecare and telehealth are viable solutions. However, it remains necessary to measure and document the exact benefits for individuals (plus their friends, family, and informal carers), for care providers and the way care is delivered, and for the wider economy. This information will ultimately demonstrate the value of telecare as a sustainable solution that supports the aging population. Several recent telecare pilots and programs have begun to tackle the issue of mainstreaming telecare services and also demonstrating their impact.

\section{UK}

In recent years, social care authorities in the UK have been putting into place telecare sensor services, eg, detectors for smoke, heating, and flooding. The UK is on the verge of taking telecare into the mainstream, driven mainly by policy and funding. Government grants have been integral in moving telecare services nearer to the mainstream.

Preventative Technology Grants ${ }^{46}$ were given to councils in England with the expectation that they develop telecare services in conjunction with partners in the NHS (National Health Service), housing and local authorities, voluntary and independent sectors, plus service users and carers. Grants to the value of $£ 80$ million (US\$107 million) were allocated over 2 years from 2006 as part of the government's commitment to modernizing and transforming care services provided by local authorities and the NHS. The grants aimed to increase the numbers of people who benefit from telecare, with a target of reaching at least 160,000 older people nationally. Some organizations have used the funding to run ongoing small-scale pilot studies. At the other end of the scale, there has been a trend toward phased mainstreaming of telecare to support health, social care, and housing services. It has been reported that there were nearly 150,000 new telecare users in England in 2006-2007, with a further 161,000 in $2007-2008 .{ }^{46}$ If all of these people benefited from enhancements to basic social alarms through addition of telecare equipment, this would amount to about $3 \%$ of the population aged 65 years or older having telecare.

In Scotland, the government has been promoting telecare service provision since 2006 through a Telecare Development program. Local care partnerships have begun to develop, extend, and mainstream telecare solutions according to individual care needs, profiles, and priorities. A Scottish strategy set out the aim that by 2010, telecare services (not counting community alarms) would be available to 75,000 people across Scotland. ${ }^{47}$ The aim was that an extra 19,000 people would be able to continue living at home. An independent evaluation of the Scottish Telecare Development program has analyzed impacts on factors such as quality of life, carers, and hospital/care admissions. ${ }^{41}$

The Welsh telecare strategy was launched in 2005 to fund local authorities. A telecare capital grant of $£ 9$ million (US\$14 million) was allocated, with a target of providing telecare equipment to 10,000 homes. Additional funding was also provided to support the development of telecare..$^{48}$ All 22 Welsh local authorities have now produced ambitious strategies for telecare. Based on monitoring reports ${ }^{48}$ it is expected that by the end of the grant period, some 45,000 people will be using a telecare service other than a community alarm - about $7 \%$ of the population aged 65 years and over.

In Northern Ireland, the Minister for Health, Social Services and Public Safety announced that $£ 1.5$ million (US\$2.4 million) would be allocated from 2008 to 2010 for pilot projects that promoted the development of new technologies to help people to live at home. ${ }^{49}$

Numerous pilot studies of telecare have been conducted in the UK, but the largest so far has been the Whole System Demonstrator program. This was undertaken in three locations in England, involving over 6,000 people and over 230 primary health care practices. It served as a randomized controlled trial covering both telecare and telehealth..$^{27,28}$ However, the results of the program suggest that the benefits of telehealth may not be clear cut and may depend on the context in which telehealth and telecare are delivered.

Also in the UK, the Assisted Living Innovation Platform has promoted the development of telecare technologies. Its successor program DALLAS ${ }^{29}$ is aiming to evaluate telecare technologies with around 169,000 users of assisted and independent living services. A key focus of this program is the evaluation of independent living technologies and services being rolled out at scale across the UK. As such, it is examining outcome-based measures for health and wellbeing as well as evaluating the barriers and facilitators for large-scale deployment of technology in practice. Crucially, this program 
is not a randomized controlled trial by design. Rather, it will report on the pragmatic issues associated with designing these services for consumers, and integrating these services into the lives of many consumers between 2012 and 2015. At the heart of this program is the development of a pragmatic evaluation framework looking at how to best document the health, wellbeing, lifestyle, and economic benefits of telecare and telehealth.

\section{Europe}

Across Europe, the need for robust evaluations has also been recognized. The Ambient Intelligence program has supported many telecare pilots in Europe. European governments have also come together in the Europe-wide research AAL program (Ambient Assisted Living) that has been running since $2007 .{ }^{30}$ In the framework of this program, participating countries, together with the European Commission, have committed to spend about $€ 50$ million (US\$67 million) per year between 2008 and 2013 on research in this field. The European Centre for Connected Health was established to promote improvements in care through the use of technology and to fast track new products and innovations in health and social services.

In 2006, the German government launched a research program on "Ageing Related Support Systems for Healthy and Independent Living." ${ }^{50}$ This research program has a budget of $€ 30$ million (US $\$ 40$ million). It emphasizes the importance of exploiting modern mainstream technologies for enabling older people who are in need of support to continue living in their own homes.

In Denmark, passive telecare systems (enhancements to basic social alarms with the addition of extra sensors) have been available for a long time (eg, the PERSONA [Perceptive Spaces Promoting Independent Aging $]^{51}$ and DREAMING ${ }^{52}$ projects). However, these systems do not seem to have been widely used. There has apparently been some resistance to passive monitoring because of the "surveillance" aspect. As a result, there is now legislation in Denmark that regulates the circumstances in which it may be used.

In the Netherlands, uptake of telecare services is estimated to be below $1 \%$ of the population aged 65 or over. Examples of telecare pilot projects in the Netherlands include "Social Alarm Plus," run by Tunstall Group PLC along with two housing associations, a call center, and a home care organization. ${ }^{53}$ This pilot involved tests with personal safety alarm systems that included an intruder alarm, a smoke alarm, an electronic door lock, and automatic lighting. A total of 120 households in the Osdorp area of Amsterdam took part, and the system was continued after the end of the project. Other housing associations in the Amsterdam region have started to show interest in experimenting with similar schemes. ${ }^{53}$

In Spain, France, and Italy more advanced telecare systems have been mainstreamed to some extent under existing social alarm schemes. ${ }^{53}$ There is no quantitative evidence available on the uptake of telecare in Italy, although it seems likely that levels of implementation are still very low. In Finland, there is no formal telecare system, although the capacity is in principle available across the country through social alarms. ${ }^{53}$

In general, telecare services in Europe seem to be mainly provided on the basis of the social alarm infrastructure. Usage levels are still low, and there have been mostly small-scale pilots and trials. Larger and more pragmatic deployments and evaluations will be needed to truly integrate telecare services into the way care is managed and provided for an aging population in Europe.

\section{Worldwide}

In the United States, there has been increasing interest in telecare, with the emphasis and focus more on health care than on social care in a wider sense. Such "telecare" services are provided by a range of organizations including medical/ clinical practices, hospitals, and social service providers both public and private. The availability of services varies from state to state, with little or no coherence in application or utilization. There is no true data available on the extent of uptake, although it seems to vary a lot across the country.

To date, the Veterans Administration health care system seems to be the main provider of telecare services with an independent living focus, even though the main emphasis of remote support and monitoring has so far been on telehealth. ${ }^{31}$ Some telecare services have been mainstreamed in the USA. In Florida, for example, LAMP (Low ADL Monitoring Program $)^{54}$ is a community care coordination service that addresses the needs of veterans for support with ADLs. This project is employing care coordination, home monitoring, and communications technology.

Japan faces a particularly severe challenge due to a high (and growing) proportion of older people. Mainstreaming of telecare in Japan has yet to occur, although some trials have been reported in recent years. A number of pilot implementations have, for instance, been funded by the Ministry of Health and Welfare. As an example, the Promotion Model project $^{32}$ is a large-scale attempt to support municipalities in adopting a set of 20 telecare services designed for older people living in the community. Previously, the Ministry had 
issued a report on "Guidelines for Implementing Information Technology in the Areas of Health, Medical Care and Welfare." Despite such efforts and the existence of a strong industrial base when it comes to equipment manufacturing, uptake of more advanced telecare applications has fallen below expectations in Japan. However, some local mainstreaming has been reported of passive alarm sensors that do not need to be actively triggered by service users. Despite widespread and strong support for telecare, the results so far have been fragmentary. ${ }^{32}$

Globally, many of the evaluations that have been reported are still considered to be pilot studies. The potential for mainstream delivery of telecare has therefore not been fully realized or evaluated so far. One success story is the SOPHIA service in Germany that was developed from a publicly funded project. ${ }^{50}$ It has been successfully mainstreamed and is currently being deployed in different parts of Germany by means of a franchise model. The DALLAS program in the UK is also looking at mainstreaming and consumer models of care across the UK, as well as examining how best to evaluate the true impact of telecare in practice. ${ }^{29}$ The results of the DALLAS evaluation will be available in June 2015.

The value of telecare technology has been long recognized in many countries. The last decade, however, has seen a shift toward evaluating these technologies and services at scale and in people's homes. It has become increasingly important to evaluate what actually happens when these services are rolled out, and when they are integrated into social and health care delivery. Another key focus is on developing pragmatic evaluation frameworks that look at not only the usability of single devices or pilot studies but also the impact on health outcomes, wellbeing, lifestyle, and the economy.

\section{Conclusion}

\section{Summary}

The world population is aging, with significant percentages of older people expected in the coming decades. It will become necessary for older people to live for longer in their own homes. Telecare using computer-based systems for social care at home has been widely accepted as an important part of the solution. Social care and health care are increasingly being integrated and supported by common technologies. Consumer-centric models of care are being adopted. People are being encouraged to take their health and wellbeing into their own hands by exploiting telecare solutions.

Telecare is provided through a home hub that links sensors, actuators, and external services to provide a range of care services in the home. Telecare technologies have progressed through three generations, from reactive to proactive to integrated. A telecare system is linked through landline, broadband, or cellular network connections to social and health care providers in the outside world. The range of sensors and actuators used in telecare continues to grow, with recent advances supporting user mobility and various sensory modalities. Telecare services are able to support aspects such as safety, security, behavior, communication, entertainment, and home automation.

Most developed countries have active telecare programs. Examples have been drawn from the UK, Europe, USA, and Japan. End users and their informal carers benefit from the improved peace of mind that telecare brings. Professional care providers gain from reduced routine monitoring and lower costs. However, telecare will require integration into daily care practices, and will require a new generation of practitioners trained in telecare technologies.

The way telecare is being designed, developed, and marketed is changing. Solutions are being developed with and for users, allowing seamless integration into their homes and lives. Telecare solutions can now be personalized for the needs, capabilities, and preferences of users - with adaptation over time as care needs evolve. Attitudes toward telecare are also changing. For example, many approaches are now combining health, wellbeing, and lifestyle technologies into off-the-shelf solutions that monitor activity in the home or while on the move, helping users to self-manage a long-term condition.

The potential benefits of telecare and assisted living have been well established. Appropriate design and deployment of telecare can improve health outcomes and quality of life, can increase independence, and can empower people and allow them to self-manage their own health and wellbeing. Telecare also offers the opportunity to be more proactive about detecting potential problems, reducing hospitalization or a decline in health. The large-scale uptake of telecare has not, however, been realized yet. This is in part due to the attitudes and expectations of end users (older adults, for example) as well as of health and social care professionals. More work is needed on user-centered design and development of consumer-ready devices, applications, and services. These must fit into homes and lifestyles and not be stigmatizing. As these devices and services become more attractive to the consumer, in terms of esthetics and cost-benefits, then telecare will become an integral part of our lives as we grow old independently in our own homes. 


\section{Future prospects}

Although significant progress has been made in telecare, further work is still required in areas such as the following.

\section{Design, usability, and user experience}

User-centered design is vital in making home care technologies acceptable and usable. Further research is needed to establish good design principles for telecare. It will also be necessary to develop interfaces to home systems for nontechnical users, allowing people to define how technology should look, behave, and adapt to them. Although the focus of this article has been on home-based care, future care should be extended to the workplace and, more generally, while users are away from home. It is likely that the smart phone will become the preferred solution for collecting and analyzing care and lifestyle data, and for providing care support. Body sensor networks are also a promising development for home and mobile use. In future, sensors may take the form of implants that monitor vital signs and everyday activity. New advances will be needed in techniques for interpreting and visualizing data, ie, in turning raw data into usable information.

\section{Reusability and personalization}

Telecare is still a relatively recent development, so solutions are often single-purpose and specialized. In fact, many of the capabilities required for home care can be supported by general-purpose components. Value can be added by creating smart services using simple devices. It is to be hoped that future home care technologies will be reusable and generalpurpose in this kind of way. User needs vary widely and can also change over time. It will therefore be necessary for future home care systems to be customizable for individual users and to adapt as their needs evolve. This will require research into how home systems can learn about the user and what their care needs are. It will also be important to make telecare services easily modifiable.

\section{Integration and interoperability}

Telecare and telehealth have evolved separately from mainstream social care and health care. As the technologies mature, it will be necessary to integrate them into daily professional practice. A current barrier to integration is the lack of technical standards for telecare. Interoperability and data sharing will require substantial effort on standards that allow interchange across manufacturers. Social work and health services have traditionally maintained separate records of care. Given the interplay between social and health care, it will be important in future to link all care records irrespective of their origin.

\section{Technology}

The tendency so far has been to focus on devices. In future, the emphasis will need to be on platforms as integrated and extensible frameworks that work with a wide variety of sensors, actuators, and services. An open question is how home care solutions should be certified. Of course, this will require standards to test against in the first place. As standards for telecare emerge, it will be necessary to decide which organizations should certify compliance.

\section{Services}

Care providers will need to embody home care technologies into their overall service provision. Ideally, care should be provided holistically, irrespective of whether the issues are social or medical. Social care and health care are separately funded in many countries. However, this can mean that the benefits of telecare (funded from the social work budget) are reaped by the health authorities (eg, due to reduced bed occupancy and readmissions). This suggests that holistic funding of care should be considered in future and not just holistic provision of care. A balance will also need to be struck between the roles of public and private telecare. In countries like the UK, the provision of care has historically been seen as a government responsibility. In contrast, care is mostly handled by the private sector in countries like the USA.

\section{Awareness and education}

Many care professionals were trained at a point that telecare did not exist or was just emerging. To make telecare effective, much more work will be needed on practitioner training, on assessments that include technological solutions, on procedures for monitoring and dealing with care data, and in development of call centers for example. Even now, few universities and colleges offer courses or degrees that focus on telecare. Although there is a rich research literature on telecare and related topics, this tends to be oriented toward the academic community. Hopefully, accessible information, practical guidelines, and procedural standards will be published in the near future for the benefit of care professionals. Public awareness of care technologies is also desirable to fuel demand for telecare products and services.

\section{Acknowledgments}

Some of the information in this article was developed by the authors on the MATCH project ${ }^{37}$ and reported among the project results. ${ }^{6}$ The MATCH project was supported by the Scottish Funding Council under grant HR04016. 


\section{Disclosure}

The authors report no conflicts of interest in this work.

\section{References}

1. Select Committee on Economic Affairs. Aspects of the Economics of an Ageing Population. London: Stationery Office Limited; 2003.

2. Carone G, Costello D. Can Europe afford to grow old? Finance and Development Magazine. 2006;43(3):8-31.

3. Katz S. Assessing self-maintenance: activities of daily living, mobility, and instrumental activities of daily living. J Am Geriatr Soc. 1983;31(12):721-727.

4. Scottish Executive. Guidance on Single Shared Assessment of Community Care Needs, Circular CCD 8/2001. Edinburgh: Scottish Government; 2001.

5. Turner KJ, Maternaghan C. Home care systems. In: Turner KJ, editor. Advances in Home Care Technologies: results of the MATCH project. Amsterdam: IOS Press; 2012:21-29.

6. Turner KJ, editor. Advances in Home Care Technologies: results of the MATCH project. Amsterdam: IOS Press; 2012.

7. Munstermann M, Stevens T, Luther W. A novel human autonomy assessment system. Sensors. 2012;12(6):7828-7854.

8. Doughty K, Cameron K, Garner P. Three generations of telecare of the elderly. J Telemed Telecare. 1996;2(1):71-80.

9. European Telecommunications Standards Institute. Telecare In and Outside Intelligent Homes - Issues and Recommendations for End User Aspects, STF264. Sophia Antipolis: European Telecommunications Standards Institute; 2012.

10. Continua Health Alliance. Connected health standards. http://www. continuaalliance.org. Accessed Apr 2013.

11. Wikipedia [homepage on the Internet]. X10 Industry Standard. Available from: http://en.wikipedia.org/wiki/X10_(industry_standard). Accessed Apr 2013.

12. UPnP Form. Universal Plug and Play. http://www.upnp.org. Accessed Apr 2013.

13. Institution of Electrical and Electronics Engineers. Local and Metropolitan Area Network Standards - Carrier Sense Multiple Access with Collision Detection (CSMA/CD) Access Method and Physical Layer Specifications, IEEE 802.3. New York: IEEE Press; 1984.

14. Bluetooth Special Interest Group. Bluetooth technology. http://www. bluetooth.com. Accessed Apr 2013.

15. ZigBee Alliance. ZigBee technology. http://www.zigbee.org. Accessed Apr 2013.

16. Yang G-Z. Body Sensor Networks. Berlin: Springer; 2006.

17. Magill EH, Blum JM. Personalised ambient monitoring: supporting mental health at home. In: Turner KJ, editor. Advances in Home Care Technologies: results of the MATCH project. Amsterdam: IOS Press; 2012:67-85.

18. McGee-Lennon MR, Gray PD, Brewster SA. Multimodal interaction and technologies for care at home. In: Turner KJ, editor. Advances in Home Care Technologies: results of the MATCH project. Amsterdam: IOS Press; 2012:106-117.

19. OSGi Alliance. OSGi System. http://www.osgi.org. Accessed Apr 2013.

20. Gil NM, Hine NA, Arnott JL, et al. Data Visualisation and Data Mining Technology for Supporting Care for Older People: Proceedings of the 9th International Conference on Computers and Accessibility. New York: ACM Press; 2007:139-146.

21. Barger TS, Brown DE, Alwan M. Health-status monitoring through analysis of behavioural patterns. IEEE Trans Syst Man Cybern A Syst Hum. 2005;35(1):22-27.

22. Lorig KR, Halsted R, Holman MD. Self-management education: history, definition, outcomes, and mechanisms. Ann Behav Med. 2003;26(1):1-7.
23. Kearney N, McCann L, Norrie J, et al. Evaluation of a mobile phonebased, advanced symptom management system (ASyMS) in the management of chemotherapy-related toxicity. Support Care Cancer. 2009; 17(4):437-444.

24. Intille SS. A new research challenge: persuasive technology to motivate healthy aging. IEEE Trans Inf Technol Biomed. 2004;8(3):235-237.

25. Alwan M, Wiley D, Nobel J. State of Technology in Ageing Services. Washington, DC: Center for Aging Services Technologies; 2007.

26. Bowes AM, McColgan G, Bell DNF. Smart Technology and Community Care for Older People: innovation in West Lothian Scotland. Edinburgh: Age Concern; 2006.

27. Steventon A, Bardsley M, Billings J, et al. Effect of telehealth on use of secondary care and mortality: Findings from the Whole System Demonstrator cluster randomised trial. BMJ. 2012;341(7862): 1756-1833.

28. Cartwright M, Hirani SP, Rixon L, et al. Effect of telehealth on quality of life and psychological outcomes over 12 months (Whole Systems Demonstrator telehealth questionnaire study): Nested study of patient reported outcomes in a pragmatic, cluster randomised controlled trial. BMJ. 2013;346(7897):f653.

29. Technology Strategy Board [homepage on the Internet]. DALLAS Delivering Assisted Living Lifestyles at Scale. Available from: http:// www.innovateuk.org/content/competition/dallas-delivering-assistedliving-lifestyles-at-sc.ashx. Accessed Apr 2013.

30. European Commission. Ambient Assisted Living Joint Programme. Available from: http://www.aal-europe.eu. Accessed Apr 2013.

31. Darkins A, Ryan P, Kobb R, et al. Care coordination/home telehealth: the systematic implementation of health informatics, home telehealth, and disease management to support the care of veteran patients with chronic conditions. Telemed J E Health. 2008;14(10):1118-1126.

32. Fujimoto M, Miyazaki K, von Tunzelmann N. Complex systems in technology and policy: Telemedicine and telecare in Japan. Telemed Telecare. 2009;15(6):175-181.

33. Beale S, Truman P, Sanderson D, Kruger J. The initial evaluation of the Scottish telecare development program. J Technol Hum Serv. 2010;28(1-2):60-73.

34. Hine NA, Stewart NM, Arnott JL, et al. Supporting the dialogue of care. In: Turner KJ, editor. Advances in Home Care Technologies: results of the MATCH project. Amsterdam: IOS Press; 2012:183-202.

35. Marschollek M, Wolf KH, Plischke M, et al. People's perceptions and expectations of assistive health-enabling technologies: an empirical study in Germany. Assist Technol. 2009;21(2):86-93.

36. Sorell T, Draper H. Telecare, surveillance, and the welfare state. Am J Bioethics. 2012;12(9):36-44.

37. MATCH Consortium [homepage on the Internet]. MATCH (Mobilising Advanced Technologies for Care at Home). Available from: http:// www.match-project.org.uk. Accessed Apr 2013.

38. Clark JS, McGee-Lennon MR. A stakeholder-centred exploration of the current barriers to the uptake of home care technology in the UK. Assist Technol. 2011;5(1):12-25.

39. Oudshoorn N. Telecare Technologies and the Transformation of Healthcare. Basingstoke: Palgrave Macmillan; 2011.

40. Taylor K, Yadav A. Telecare and Telehealth - A Game Changer for Health and Social Care. London: Deloitte Centre for Health Solution; 2013.

41. Beale S, Sanderson D, Kruger J. Evaluation of the Telecare Development Programme: Final Report. Edinburgh, UK: Scottish Government; 2009. Available from: http://www.jitscotland.org.uk/downloads/1235404195B59058 Final Report low res.pdf. Accessed Apr 2013.

42. MultiMemoHome Consortium. The MultiMemoHome project. http:// www.multimemohome.com. Accessed May 2013.

43. McGee-Lennon MR, Smeaton A, Brewster SA. Designing home care reminder systems: lessons learned through co-design with older users. In: 6th International Conference on Pervasive Computing Technologies for Healthcare. New York: IEEE Press; 2012.

44. COBALT Consortium. The COBALT project. Available from: http:// cobaltproject.org. Accessed Jun 2013. 
45. Fisk M. Telecare equipment in the home: Issues of intrusiveness and control. Telemed Telecare. 1997;3(1):30-32.

46. UK Government. Building Telecare in England. Available from: http:// webarchive.nationalarchives.gov.uk/20130107105354/http://www. dh.gov.uk/en/Publicationsandstatistics/Publications/Publications PolicyAndGuidance/DH_4115303. Accessed September 3, 2013.

47. Scottish Government. An Assessment of the Development of Telecare in Scotland 2006-2010. Available from: http://www.scotland.gov.uk/ Resource/Doc/328586/0106225.pdf. Accessed May 2013.

48. Welsh Assembly. The Welsh Telecare Strategy. Available from: http:// www.ssiacymru.org.uk/telecare. Accessed Jun 2013.

49. HealthTech and Medicines Knowledge Transfer Network. Northern Ireland. Available from: http://www.healthktn.org/capabilitymap/ northern-ireland.html. Accessed Sep 2013.
50. SOPHIA Consortium. The SOPHIA Service in Germany. Available from: http://www.sophia-franken.de. Accessed Apr 2013.

51. PERSONA Consortium. The PERSONA Project. Available from: http://cordis.europa.eu/projects/rcn/80532_en.html. Accessed Sep 3, 2013.

52. DREAMING Consortium. The DREAMING Project. http://ec.europa. eu/information_society/apps/projects/factsheet/index.cfm?project_ $\mathrm{ref}=225023$. Accessed Sep 2013 .

53. Empirica GmbH. ICT and Ageing - European Study on Users, Markets and Technologies. Available from: http://www.ict-ageing.eu. Accessed May 2013.

54. US Department of Veteran Affairs [homepage on the Internet]. Low ADL Monitoring Program (LAMP). http://www.northflorida.va.gov/ NORTHFLORIDA/services/lamp.asp. Accessed Apr 2013.

\section{Publish your work in this journal}

Smart Homecare Technology and TeleHealth is an international, peer-reviewed, open access online journal publishing original research, reviews, editorials and commentaries on the application of technology to support people and patients at home and in assisted living centers to optimize healthcare and management resources. Specific topics in the journal include: Development and application of devices within the home and embedded in appliances; Healthcare provider communication and education tools; and drug ordering and adherence. The manuscript management system is completely online and includes a very quick and fair peer-review system, which is all easy to use. Visit http://www.dovepress.com/ testimonials.php to read real quotes from published authors. 